\title{
Quantum Gates Based on Polarization Transfer NMR Spectroscopy
}

\author{
A. GüleÇ AND S. BAHÇElí \\ Department of Physics, Faculty of Arts and Sciences \\ Süleyman Demirel University, Isparta, Turkey
}

(Received January 31, 2005; in final form March 16, 2005)

\begin{abstract}
We first introduce an alternative way to study the insensitive nuclei enhanced by polarization and distortionless enhancement by polarization transfer NMR experiments with the product operator descriptions for weakly coupled $I S(I=1 / 2, S=1 / 2)$ spin systems as a quantum controlled not gate and a not gate, respectively. In this framework we apply the density matrix operators obtained from the analytical descriptions of the mentioned pulse sequences by using product operator formalism step by step on the NMR spin product states represented in Dirac's notation.
\end{abstract}

PACS numbers: 82.56.Dj, 82.56.Jn

\section{Introduction}

The starting point for the journey through the fascinating realm of computers can be based on a mathematical analysis of logic presented by George Boole who published a classical book An Investigation of the Laws of Thought on Which Are Founded the Mathematical Theses of Logic and Probabilities in 1854. Traditional digital computers operate using two values of a bit, 0 and 1, in Boolean algebra. Later these two values are used as quantum bits (or qubits) in quantum computers [1]. In 1982 Feynman suggested that quantum mechanical systems have a very large information processing abilities [2]. Thus a new class of computers which are based on the NMR properties of some molecules has been of great scientific interest [3-6]. In this framework Freeman and co-workers first implied the use of the insensitive nuclei enhanced by polarization transfer (INEPT) pulse sequence as a logic gate in NMR quantum computing [7]. Later the conventional INEPT

*corresponding author 
NMR pulse sequence was studied as a controlled-not (briefly CNOT or CN) gate for the spin- $1 / 2$ nuclei by using the similarities between the unitary operators of CNOT gate and INEPT pulse sequence [8]. On the other hand, the INEPT NMR experiment is a well-known pulse sequence and is usually described by the product operator theory as a simple quantum mechanical method [9-13]. In a similar way the distortionless enhancement by polarization transfer (DEPT) pulse sequence and its analytical descriptions by using product operator formalism for weakly coupled $I S(I=1 / 2, S=1 / 2 ; 3 / 2)$ spin system has been widely investigated [14-17].

The main problem in the NMR quantum computing computers is to create the pure states (or pseudopure states) from the mixed states of the system under studied as the inputs of the quantum gates. However some methods were suggested for the creation of pseudopure states in the $I \geq 1 / 2$ systems $[18,19]$.

This work presents an alternative way in order to perform the conventional INEPT and DEPT pulse sequences described by using product operator theory for weakly coupled $I S(I=1 / 2, S=1 / 2)$ spin systems as a $C N$ gate and a NOT gate, respectively. For this purpose the density matrix operators obtained from the analytical descriptions of the mentioned pulse sequences by using product operator formalism for weakly coupled $I S(I=1 / 2, S=1 / 2)$ spin systems have been applied step by step on the NMR spin product states represented in Dirac's notation.

\section{The applications of the density operators for the INEPT pulse sequences to the pseudopure states of the $I S(I=1 / 2, S=1 / 2)$ spin systems}

The pulse sequence of the INEPT NMR experiment for weakly coupled IS $(I=1 / 2, S=1 / 2)$ spin systems is given in Fig. 1 . The eigenfunctions of a such system can be written in Dirac's notation as follows:

$$
\begin{array}{ll}
|\alpha \alpha\rangle=\left|\frac{1}{2}, \frac{1}{2}\right\rangle=|++\rangle=|00\rangle, \quad|\beta \alpha\rangle=\left|-\frac{1}{2}, \frac{1}{2}\right\rangle=|-+\rangle=|10\rangle, \\
|\alpha \beta\rangle=\left|\frac{1}{2},-\frac{1}{2}\right\rangle=|+-\rangle=|01\rangle, \quad|\beta \beta\rangle=\left|-\frac{1}{2},-\frac{1}{2}\right\rangle=|--\rangle=|11\rangle .
\end{array}
$$

Each eigenstate given in Eq. (1) can be regarded as the inputs of a quantum gate $\mathrm{CN}$ with two qubits. On the other hand, the density matrix operators of INEPT pulse sequence in Fig. 1 for a weakly coupled $I S(I=1 / 2, S=1 / 2)$ spin system by using product operator formalism are well known [10]. We consider the chloroform $\left({ }^{1} \mathrm{H}^{13} \mathrm{CCl}_{3}\right)$ as a suitable molecule for $I S\left({ }^{1} \mathrm{H}=I=1 / 2,{ }^{13} \mathrm{C}=\right.$ $S=1 / 2)$ spin system. Since the gyromagnetic ratios of the proton and carbon atoms are $\gamma_{\mathrm{H}}=26.75 \times 10^{7} \mathrm{~T}^{-1} \mathrm{~s}^{-1}$ and $\gamma_{\mathrm{C}}=6.73 \times 10^{7} \mathrm{~T}^{-1} \mathrm{~s}^{-1}$, respectively, we take $\alpha=\gamma_{\mathrm{C}} / \gamma_{\mathrm{H}}=0.25$ in our calculations. The density matrix operators 


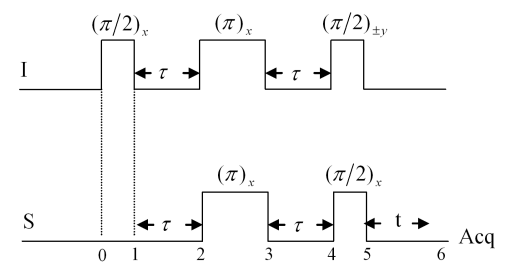

Fig. 1. The pulse sequence of INEPT NMR spectroscopy $\left(\tau=\frac{1}{4 J}\right)$.

calculated for $I S(I=1 / 2, S=1 / 2)$ spin system by using these numerical values are given in Table I.

TABLE I

For $I S\left({ }^{1} \mathrm{H}=I=1 / 2,{ }^{13} \mathrm{C}=S=1 / 2\right)$ spin system, the calculated density matrix operators in INEPT pulse sequence.

\begin{tabular}{l}
\hline \hline$\sigma_{0}=I_{z}+0.25 S_{z}, \quad \sigma_{1}=-I_{y}+0.25 S_{z}$, \\
$\sigma_{2}=-\frac{\sqrt{2}}{2} I_{y}+\sqrt{2} I_{x} S_{z}+0.25 S_{z}, \quad \sigma_{3}=\frac{\sqrt{2}}{2} I_{y}-\sqrt{2} I_{x} S_{z}-0.25 S_{z}$, \\
$\sigma_{4}=-2 I_{x} S_{z}-0.25 S_{z}, \quad \sigma_{5}=-2 I_{z} S_{y}+0.25 S_{y}, \quad \sigma_{6}=2 S_{y} S_{I} S_{J}^{\prime}$ \\
\hline where $S_{I}=\sin \left(\Omega_{I} t\right)$ and $S_{n J}^{\prime}=\sin (n \pi J \tau)$.
\end{tabular}

Let us apply the density matrix operators given in Table I to the state $|\alpha \alpha\rangle=\left|\frac{1}{2}, \frac{1}{2}\right\rangle=|++\rangle=|00\rangle$ sequently. The results obtained are as follows:

$$
\begin{aligned}
& \sigma_{0}|++\rangle \Rightarrow 0.63|++\rangle, \\
& \sigma_{1}(0.63|++\rangle) \Rightarrow-0.32 \mathrm{i}|-+\rangle+0.08|++\rangle, \\
& \sigma_{2}(-0.32 \mathrm{i}|-+\rangle+0.08|++\rangle) \Rightarrow(0.122-0.112 \mathrm{i}|++\rangle \\
& \quad+(-0.068 \mathrm{i}+0.028)|-+\rangle, \\
& \quad \sigma_{3}[(0.122-0.112 \mathrm{i})|++\rangle+(-0.068 \mathrm{i}-0.028)|-+\rangle] \\
& \quad \Rightarrow(0.09 \mathrm{i}-0.007)|-+\rangle+(-0.048+0.028 \mathrm{i})|++\rangle, \\
& \sigma_{4}[(0.09 \mathrm{i}-0.007)|-+\rangle+(-0.048+0.028 \mathrm{i})|++\rangle] \\
& \quad \Rightarrow(-0.056-0.048 \mathrm{i})|++\rangle+(0.0249-0.0253 \mathrm{i})|-+\rangle, \\
& \sigma_{5}[(-0.056-0.048 \mathrm{i})|++\rangle+(0.0249-0.0253 \mathrm{i})|-+\rangle] \\
& \quad \Rightarrow(0.021 \mathrm{i}-0.018)|+-\rangle+(0.009 \mathrm{i}+0.016)|--\rangle,
\end{aligned}
$$




$$
\begin{aligned}
& \sigma_{6}[(0.021 \mathrm{i}-0.018)|+-\rangle+(0.009 \mathrm{i}+0.016)|--\rangle] \\
& \quad \Rightarrow(0.018 \mathrm{i}+0.021)|++\rangle+(-0.016 \mathrm{i}+0.009)|-+\rangle \\
& \quad \cong 0.021|++\rangle \cong 0.021|00\rangle,
\end{aligned}
$$

where the terms with the real and biggest coefficient have been taken account as the result. In the last step we consider the term $S_{I}$ seen in Table I as a certain value. In a similar way the calculations can be repeated for the remains of eigenstates given in Eq. (1) and the results obtained can be listed by considering the truth table of quantum $\mathrm{CN}$ gate as seen in Table II.

TABLE II

The behaviour of the INEPT NMR experiment for a weakly coupled IS $(I=1 / 2, S=1 / 2)$ spin system as a quantum $\mathrm{CN}$ logic gate.

\begin{tabular}{c|c|c|c}
\hline \hline \multicolumn{2}{c|}{ Inputs } & \multicolumn{2}{c}{ Outputs } \\
\hline INEPT & CN & $U_{\text {INEPT }}$ & CN \\
\hline$|++\rangle$ & $|00\rangle$ & $0.21|++\rangle$ & $|00\rangle$ \\
$|+-\rangle$ & $|01\rangle$ & $0.015|--\rangle$ & $|11\rangle$ \\
$|-+\rangle$ & $|10\rangle$ & $0.004|-+\rangle$ & $|10\rangle$ \\
$|--\rangle$ & $|11\rangle$ & $0.009|+-\rangle$ & $|01\rangle$ \\
\hline \\
where UINEPT denotes the results \\
obtained from the output of INEPT \\
pulse sequence.
\end{tabular}

By comparing the outputs of $U_{\text {INEPT }}$ and the truth table of quantum logic gate $\mathrm{CN}$ we can express that this pulse sequence can be used as an " $S$ controlling I" CN quantum gate with two qubits [7]. As seen in Table II after the application of $U_{\text {INEPT }}$ operation to second qubit (the control bit of CN gate), if the first qubit (the target bit of $\mathrm{CN}$ gate) is $|+\rangle=|0\rangle$ in the input, it keeps its own value in output but if the control bit is in $|-\rangle=|1\rangle$ in the input, the target bit in output subjects to a not operation of its input value. This result is in a good agreement with Vadersypen and co-workers's work [8].

\section{The applications of the density operators for the DEPT pulse sequences to the pseudopure states of the $I S(I=1 / 2 ; S=1 / 2)$ spin systems}

The pulse sequence of the DEPT NMR experiment is given in Fig. 2 [14]. The density matrix operators in every single stage of this pulse sequence (denoted with 


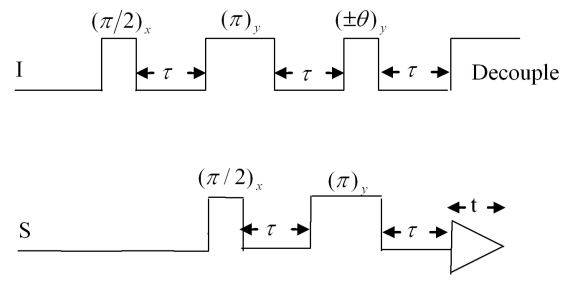

Fig. 2. The DEPT NMR pulse sequence $\left(\tau=\frac{1}{2 J}\right)$.

the numbers in Fig. 2) for a weakly coupled $I S(I=1 / 2, S=1 / 2)$ spin systems by using the product operator formalism have been well described [16]. Considering a suitable molecule containing ${ }^{13} \mathrm{C}\left\{{ }^{1} \mathrm{H}\right\}$ nuclei (for instance, the chloroform) and taking $\alpha=\gamma_{\mathrm{C}} / \gamma_{\mathrm{H}}=0.25$, the density matrix operators calculated in this pulse sequence for a weakly coupled $I S\left({ }^{1} \mathrm{H}=I=1 / 2,{ }^{13} \mathrm{C}=S=1 / 2\right)$ are given in Table III.

TABLE III

For $I\left({ }^{1} \mathrm{H}=I=1 / 2,{ }^{13} \mathrm{C}=S=1 / 2\right)$ spin systems, the calculated density matrix operators in DEPT pulse sequence.

\begin{tabular}{c|c}
\hline \hline$\sigma_{0}=0.25 S_{z}+I_{z}$, & $\sigma_{4}=-2 I_{x} S_{y}$, \\
$\sigma_{1}=0.25 S_{z}-I_{y}$, & $\sigma_{4}=\sigma_{3}$, \\
$\sigma_{2}=2 I_{x} S_{z}$, & $\sigma_{5}=2 I_{z} S_{y} \sin \theta$, \\
$\sigma_{3}=-2 I_{x} S_{y}$, & $\sigma_{6}=2 I_{z} S_{x} \sin \theta$
\end{tabular}

The applications of the density matrix operators in Table III sequently to eigenstates denoted in Dirac's notation in Eq. (1) are performed in a similar way to those of Sect. 2. For a comparison the results obtained by considering the values of the terms with only real and biggest coefficients and the truth table of the NOT logic gate which is used in the conventional and quantum computers are presented in Table IV. As seen in Table IV, the outputs of DEPT NMR pulse sequence are corresponding to a not operation of the initial states of the second qubits or the $S$ spin states. For example if the $I S(I=1 / 2, S=1 / 2)$ spin system is in the initial state $|--\rangle=|11\rangle$ the output obtained after the operation of DEPT pulse sequence is in the state $|-+\rangle=|10\rangle$. That is, the initial state of second qubit (or the $S$ spins) subjects to a NOT operation in the output while the first qubit (or the $I$ spins) can keep their states at the beginning.

Therefore it can be said that the DEPT NMR experiments perform a NOT operation for the second qubit in the systems with two qubits and behave as NOT logic gates in the quantum computers. 
TABLE IV

The behaviour of DEPT NMR experiment for a weakly coupled $I S\left({ }^{1} \mathrm{H}=I=1 / 2,{ }^{13} \mathrm{C}=S=1 / 2\right)$ spin system as a NOT logic gate.

\begin{tabular}{c|c|c|c}
\hline \hline \multicolumn{2}{c|}{ Inputs } & \multicolumn{2}{c}{ Outputs } \\
\hline DEPT & NOT gate & $U_{\text {DEPT }}$ & NOT gate \\
\hline$|++\rangle$ & $|00\rangle$ & $(-0.005) \sin ^{2} \theta|+-\rangle$ & $|01\rangle$ \\
$|+-\rangle$ & $|01\rangle$ & $(0.003) \sin ^{2} \theta|++\rangle$ & $|00\rangle$ \\
$|-+\rangle$ & $|10\rangle$ & $(0.003) \sin ^{2} \theta|--\rangle$ & $|11\rangle$ \\
$|--\rangle$ & $|11\rangle$ & $(0.005) \sin ^{2} \theta|-+\rangle$ & $|10\rangle$ \\
\hline
\end{tabular}
DEPT pulse sequence.

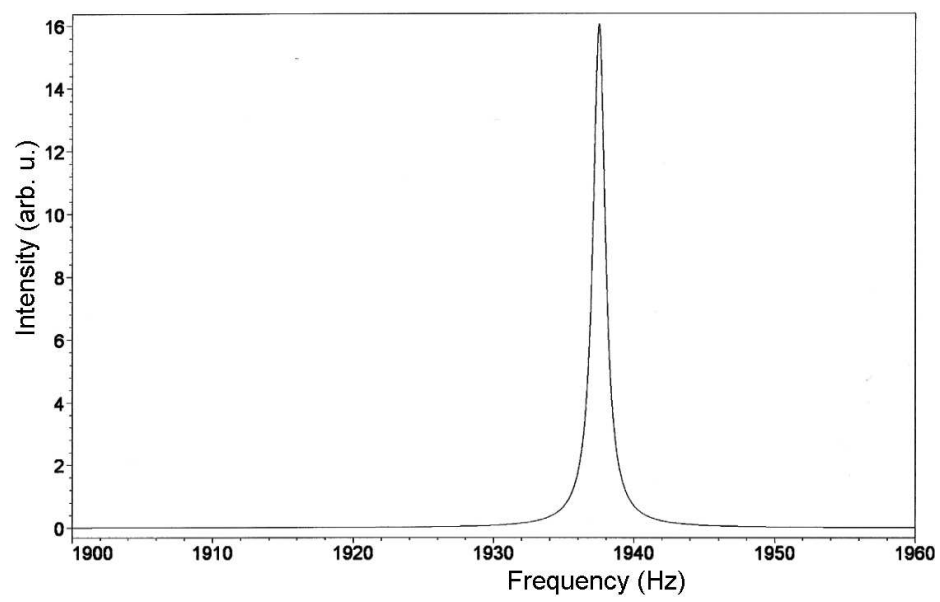

Fig. 3. The simulated ${ }^{13} \mathrm{C}$ DEPT NMR spectrum of $\mathrm{HCCl}_{3}$ for $\theta=90^{\circ}$.

On the other hand, it is well known that the angle $\theta$ or the length of ${ }^{1} \mathrm{H}$ pulse at the end of DEPT NMR pulse sequence given in Fig. 2 can be varied from $45^{\circ}$ to $135^{\circ}$ and the signal intensity depends on the angle $\theta$. The simulated ${ }^{13} \mathrm{C}$ DEPT NMR spectrum for the methine $(\mathrm{CH})$ group of ${ }^{1} \mathrm{H}^{13} \mathrm{CCl}_{3}$ (chloroform) by using the data given in Ref. [20] in the Maple programme by taking $\theta=90^{\circ}$ is given in Fig. 3.

\section{Conclusions}

As a conclusion we can state that it is obviously possible to develop this new and compact quantum mechanical method as an alternative way for the use of multipulse NMR experiments as the quantum logic gates. Furthermore we think 
that this method may be more suitable for the present algorithms with two qubits in quantum computers.

On the other hand, we predict that this alternative method can be expanded to the other weakly coupled $I S(I \geq 1 / 2, S \geq 1 / 2)$ spin system by defining their eigenstates in Dirac's notation.

\section{References}

[1] R. Landauer, IBM J. Res. Develop. 5, 183 (1961).

[2] R.P. Feynman, Int. J. Theor. Phys. 21, 467 (1982).

[3] D.G. Cory, A.F. Fahmy, T.F. Havel, Proc. Natl. Acad. Sci. 94, 1634 (1997).

[4] N.A. Grenshenfeld, I.L. Chuang, Science 275, 350 (1997).

[5] I.L. Chuang, N. Greshenfeld, M. Kubinec, Phys. Rev. Lett. 80, 3408 (1998).

[6] J.A. Jones, Science 280, 229 (1998).

[7] N. Linden, H. Barjat, E. Kupce, R. Freeman, Chem. Phys. Lett. 307, 198 (1999).

[8] L.K.M. Vandersypen, C.S. Yannoni, I.L. Chuang, in: Encyclopedia of Nuclear Magnetic Resonance, Vol. 9, Advanced in NMR, Eds. D.M. Grant, R.K. Harris, 2002, p. 687.

[9] G.A. Morris, R. Freeman, J. Am. Chem. Soc. 101, 760 (1979).

[10] O.W. Sorensen, R.R. Ernst, J. Magn. Reson. 51, 477 (1983).

[11] R. Ernst, G. Bodenhausen, A. Wokaun, Principles of Nuclear Magnetic Resonance in One and Two Dimensions, Oxford University Press, Oxford 1987.

[12] N. Chandrakumar, Basic Principles, Progress in NMR, Eds. E. Fluck, H. Günter, Vol. 34, Springer-Verlag, Berlin 1996.

[13] A. Tokatl, M. Şahin, Ö. Tezel, A. Gençten, S. Bahçeli, J. Magn. Reson. 169, 68 (2004).

[14] D.M. Doddrell, D.T. Pegg, M.R. Bendall, J. Magn. Reson. 48, 323 (1982).

[15] H. Bildsoe, S. Donstrupt, H.J. Jacobsen, O.W. Sorensen, J. Magn. Reson. 53, 154 (1983).

[16] N. Chandrakumar, S. Subramian, Modern Techniques in High Resolution FT NMR, Springer, NewYork 1982.

[17] Ö. Tezel, A. Gençten, A. Tokatlı, M. Şahin, S. Bahçeli, Acta Phys. Pol. A 104, 503 (2004).

[18] E. Knill, I.L. Chuang, R. Flamme, Phys. Rev. A 57, 3348 (1998).

[19] A.K. Khitrin, B.M. Fung, J. Chem. Phys. 112, 6963 (2000).

[20] C.S. Yannoni, M.S. Sherwood, D.C. Miller, I.L. Chuang, L.M.K. Vandersypen, M.G. Kubinec, Appl. Phys. Lett. 75, 3563 (1999). 